\title{
Minerva, have you led us astray?
}

\author{
Jan Shield
}

A debate has arisen in the pages of the $B M F$ about the wearing of bicycle helmets. The latest round opened with Minerva's piece ${ }^{1}$ on Nigel Unwin's question about what criteria must be met before helmet wearing is made compulsory. ${ }^{2}$ Unwin suggested four criteria: effectiveness, personal liberty, public acceptability, and the promotion of the health benefits of cycling. He suggests following an 'ethical map' by considering principles, precedents, and consequences. He contends that preventive strategies may do more harm than good, that perhaps prevention is not always better than cure, and cites Geoffrey Rose's answer to the question of how good the evidence must be before an intervention is promoted: 'That depends on the consequences of making the wrong decision, whether positive or negative . . $\therefore$ In other words, lacking complete evidence is alright as long as no harm is done, but where interventions are legislated, the evidence needs to be compelling and popular acceptance high. Unwin uses the distinction drawn by the utilitarian philosopher, John Stuart Mill, between actions which affect oneself and those which affect others (self regarding and other regarding actions). It's alright to kill yourself but not others. Mill recognised the difficulty of making distinctions between actions which are merely inconvenient for others and those which require punitive action, and he noted that individual liberty considerations should not be extended to children.

Unwin identifies two key principles in the debate over mandatory cycling helmets: (1) the need for a high degree of evidence of the health benefits to be expected from the intervention for those on whom it is imposed; and (2) the mandation for adults must benefit society as a whole and not just individual cyclists.

The precedents Unwin refers to are the mandatory wearing of motorcycle helmets and seat belts in the UK. After five attempts to pass legislation, seat belt wearing finally became compulsory in UK in 1981. Most of the population eventually agreed that they were effective in reducing death and injury. Here, Unwin discusses the saving to the health system of reducing the occupancy rate of 150000 bed nights a year (1981 estimates) for injuries from motor vehicle crashes. It was generally agreed that the benefits to be gained outweighed the infringement of liberty. Unwin suggests that the same is true for cyclists-there is a need for widespread agreement by a majority of cyclists, as well as the public at large, that the potential benefits of being compelled to wear helmets will outweigh the loss of freedom.

The beneficial consequences of mandatory helmet wearing, assuming they are effective in doing so, is a reduction in serious head injuries. The disadvantageous consequence is the loss of personal freedom. Unwin finds three other disbenefits: the problem of enforcement, the increased sense of security leading to greater risk taking, and that legislation might deter cycling. He does not consider the additional benefit of role modelling for children-children will perceive adult exhortations to wear bicycle helmets as hypocritical if adults do not wear helmets themselves. As well as enforcement, there is also the issue of compliance-that not everyone adheres to the law all the time. Most drivers will leave their vehicles at parking meters for longer than the stated time and stop in 'No Standing' zones from time to time without feeling they are breaking the law in a serious way. The same may well be true of cyclists. Certainly widespread support for helmets will reduce the problem of enforcement. The argument that cyclists feel safer while wearing helmets and therefore take more risks has been used to argue against helmets altogether. Therefore, the increased protection for individuals must be weighed against the possible increase in injury rates due to 'risk compensation'. Unwin argues that good data on compensatory behaviour by cyclists is needed before legislation is enacted, and that we need compelling evidence that 'helmets reduce the rate of head injuries to cyclists'. There is some evidence that compulsion to wear helmets can act as a deterrent to cycling. The benefits of cycling are twofold: health benefits to the cyclist in increased fitness, and improved quality of urban environments assuming an increase in cycling leads to a decrease in driving. Mandation must ensure that 'helmet wearing would not make the public health benefits of increased cycling harder to obtain'.

In his summing up, Unwin states that the assumption that 'the justification for enforcement rests solely on demonstrating disbenefits to others (such as avoidable use of the common resource)' is wrong. The justification, he believes, lies in the demonstration that benefits of legislation clearly outweigh the disbenefits, difficult in the absence of an objective measuring device. Unwin believes that the best scale is the 'collective judgment of the people' as expressed by elected representatives. How this might be measured is unclear since many issues affect voters' judgments of their representatives at election time. 
Her comment on Unwin's treatise elicited the information that Minerva, a keen cyclist, does not like wearing her helmet! ${ }^{1}$ This opinion drew a joint response from the sharp eyed Ronald Davis and the erudite Barry Pless who assured readers that the vast bulk of evidence supports the strong recommendation that cyclists should wear helmets and that Minerva's negative view might infect her readers. ${ }^{3}$ Davis' and Pless's letter led to a flurry of other letters, six of which were published, ${ }^{4-9}$ presumably infected by Minerva since all supported her view; none supported Davis and Pless.

Adrian Davis of the Health and Transport Research Group in Milton Keynes opined that the Davis/Pless view 'illustrates the worrying reductionist tendency in research into health promotion and illness prevention, which often results in sight being lost of the ultimate goal of promoting health'. ${ }^{4}$ Is the objective of promoting health different from promoting safety to enhance health and wellbeing? Davis uses a viewpoint similar to his own to support his argument-that expressed by statistician Dorothy Robinson in a detailed analysis of the statistical methods of some of the research. ${ }^{10}$ She also took the opportunity to write to the $B M F$ decrying the deterrent effect on cycling and citing studies conducted in New South Wales and Victoria, Australia, to demonstrate that cycling had decreased. ${ }^{6}$

Self confessed helmet wearer, Tony Waterston of Newcastle on Tyne, thought it strange that Davis and Pless had not identified the root cause of cycling injuries-motorists. ${ }^{5}$ (Many studies show that children injured while riding bicycles do not have collisions with motor vehicles but rather come off their cycles after riding on rough ground, encountering an uneven section of pavement, or losing control.) Nevertheless, Waterston's point is well made that equal time be given to the modification of motorists' behaviour by increasing the cost of petrol to reduce their 'car addiction', reducing their speed, and to environmental changes like separating bicycles from motor traffic and restricting car access to city centres. Welsh based consultant in public health medicine, Richard Keatinge, contends that 'Retailers and manufacturers of helmets are the only people likely to benefit from compulsion, which may well discourage many people from obtaining the health benefits of cycling'. This view is reinforced by the formidable Mayer Hillman who weighs into the argument with the view that 'Lowering the risk of collision in the first place is a far more effective way of preventing [head] injuries than is wearing a helmet' ${ }^{8}$ True, perhaps, but is it possible to turn back the clock to a time when you were more likely to be run down by a horse and carriage? Reducing traffic speed and volume is an admirable aim, and injury data from Denmark and the Netherlands, where cycling rates are much higher and helmet wearing rates much lower, do demonstrate lower injury rates, but that is not the case elsewhere. Bicycle helmets were designed to be protective in the event of a fall, not in the event of a collision with a motor vehicle, writes Hillman, and attributes the danger to cyclists' perception of increased safety. Hill man's final barb 'Perhaps the reputation of advocates of helmet wearing is more at risk. than the heads of cyclists who do not wea helmets' is intended to wound.

The final letter in the series, from physi cian G H Hall, claims that 'This intolerances of the do gooders is objectionable and $\vec{\Phi}$ indeed, counter productive to their aims' Hall announces an intention to continue smoking [his?] pipe and riding his bicycle helmetless.

Interestingly, none of the letter writerso referred back to the Unwin article which waș the catalyst for Minerva's comment and the Davis/Pless response. Three, however, referred to the Robinson article supporting their posi $=$ tion, although Robinson herself did not.

Are the arguments against bicycle helmetsall from disaffected cyclists who have failed to accept a technological solution to an injury? problem? A case-control study of the char $\frac{\mathbb{}}{3}$ acteristics of proponents and antagonist might be useful here! What is the common theme of their arguments and where do thesee arguments stem from? Are their arguments valid, or good examples of sophistry, personaf opinion disguised as questioning the applica tion of scientific principles to injury preven tion, or is it deeper than that? Injury? prevention and control researchers and prac $\Omega$ titioners must address these concerns. Ares they more difficult to counter when they come from our colleagues (who are presum ably better at constructing arguments) than when concerns come from the public? In some ways the arguments against wearing bicycle helmets are similar to the earlie? arguments against wearing seat belts, alludedf to by Unwin, and become less relevant withe the elapse of time. Is it just that some people haven't yet adjusted to the idea of a changing world and that the present flurry of anxiety? will die down in the 10 years or so it migho take some people to adapt? Or is it that their challenge to the dominant paradigm or the received wisdom in injury prevention isp threatening to us? I'd like to think it's a․ challenge to all of us to think more deeply and act more creatively about the conse quences of the interventions we propose anc to become more sophisticated in anticipating arguments before they are put so that our ability to counter them is not diminished bye our being caught on the back foot.

The final words belong to Thompson et al whose recently published case-control studyo shows that helmets are effective for all age groups, with overall protection of around $80 \%$ reduction in risk for head or brain injuries including those sustained in collisions with motor vehicles. ${ }^{11}$ Regarding the presumeof increase in risk taking, this study found that helmeted cyclists would have to increase theifo risk taking fourfold for the argument that helmet wearing encourages greater risk taking to hold true. 
1 Minerva. BMf 1996; 312: 1310.

Unwin NC. Cycle helmets-when is legislation justified? $\mathcal{F}$ Med Ethics 1996; 22: $41-5$

3 Davis RM, Pless B. Evidence shows that cyclists should wear helmets. BMF 1996; 313: 629.

Davis A. Increasing the number of cyclists is more important. $B M \mathcal{F}$ 1997; 314: 69.

Waterston T. Motorists are the cause of the problem. $B M \mathcal{F}$ 1997; 314: 69 .

6 Robinson DL. Australian laws making helmets compulsory deterred people from cycling. $B M \mathcal{F}$ 1997; 314: 69-70.
7 Keatinge R. Better to control the demand for fast cars. $B M \mathcal{F}$ 1997; 314: 70

8 Hillman $M$. Health benefits of cycling greatly outweigh loss of life years from deaths. BMF 1997; 314: 70.

9 Hall GH. Do gooders' intolerance is counter productive to their aims. BMF 1997; 314: 70.

10 Robinson DL. Head injuries and bicycle helmet laws. Accid Anal Prev 1996; 28: 463-75.

11 Thompson DC, Rivara FP, Thompson R. Effectiveness of bicycle safety helmets in preventing head injuries. $\mathfrak{F} A M A$ 1996; 276: $1968-73$.

\title{
Introduction to statistics-1. The confidence interval
}

\author{
Robert W Platt
}

Alert readers will note that the Instructions to Authors state 'In statistical analyses 95\% confidence intervals should be used where appropriate'. In applied statistics, it is customary to present the results of experiments in the form of $p$ values. An alternative, with much to offer, is the confidence interval. In this column, I will define the confidence interval both in mathematical terms and in layman's language, and discuss appropriate and inappropriate uses of confidence intervals.

Put simply, the confidence interval (or confidence limits) can be thought of as a range of values or interval that contains the 'true value' $95 \%$ of the time. It can be argued that in fact hypothesis testing is a poor use of statistical techniques, and does not really give a researcher the information he or she needs. ${ }^{1}$ Hypothesis tests (and $\mathrm{p}$ values) simply give information about the likelihood of a certain dataset under the null hypothesis. We are almost never interested in the null hypothesis, which usually is that there is no difference between two or several groups (or, in regression, that a dependent variable is not associated with a particular independent variable). What we are almost always interested in is the most plausible value of the true difference (or regression coefficient) and the degree of certainty in our estimation. Confidence intervals give both of these; hypothesis tests provide neither. So, in simple terms, a confidence interval can be thought of as an interval that includes the true value $95 \%$ of the time.

In statistical language, a $100(1-\alpha) \%$ confidence interval for a parameter $q$ is given by $\left(q_{1}, q_{h}\right)$ where the probability

$$
\mathrm{P}\left(\mathrm{q}_{\mathrm{l}}<\mathrm{q}<\mathrm{q}_{\mathrm{h}}\right)=1-\alpha .
$$

Thus a confidence interval is basically a formula to calculate the interval or range, which, when an experiment is repeated many times, the fraction of these intervals that cover the true value approaches $1-\alpha$. It is important to note that $\mathrm{q}$ is a fixed number-the true underlying parameter (or value)-and so it does not change and is not random. Therefore, the definitions do not refer to probability about $\mathrm{q}$, but about its limits.

Using the formula enables us to say that based on our data, the interval gives the most plausible values for the true parameter. We say 'plausible' and avoid making probability statements about $q$ because it is fixed and can't change. Remember too, that the statement depends completely on the data from your study.

What are the uses of confidence intervals and what do they allow us to do that other methods (hypothesis testing and $p$ values) don't? First, unlike a hypothesis test, the confidence interval provides a measure of location-it gives the most plausible estimate of the true parameter, given the observed data. Second, it can be used to gauge significanceif a $95 \%$ confidence interval excludes the value 0 we conclude that 0 is not a likely value of the true parameter. Finally, and perhaps most importantly, a confidence interval gives information about the certainty of estimates and the amount of unexplained variability. A wide confidence interval implies a lot of unexplained variability and thus much uncertainty. In hypothesis testing, a small $\mathrm{p}$ value indicates a large effect, low variability, or both, but it is not self evident which is most important. Confidence intervals transparently give this information by showing the effect size AND the uncertainty.

Confidence intervals can also be used when designing a study to choose appropriate sample sizes. In the same way that you can calculate the sample size for a given power level for a certain effect size, you can also calculate the sample size for a given confidence interval width at a certain effect size, and hence specify the required level of precision.

In sum, there are many advantages to presenting data using confidence intervals, and some drawbacks to simply reporting $p$ values. In addition, confidence intervals are easily calculated and available as options with most software packages. For these reasons, I recommend they be used routinely.

\section{References and further reading}

1 Oakes M. Statistical inference. Chestnut Hill, MA: Epidemiology Resources Inc, 1986

2 Gardner MJ, Altman DG. Statistics with confidence. London, UK: BMJ, 1989.

3 Swinscow TDV. Statistics at square one. London, UK: BMJ, 1980 